\title{
Synthesis of microcrystalline cellulose from pretreated cotton obtained from Bombax ceiba $L$. and its characterization
}

\author{
S. M. Haque ${ }^{1}$, A. A. Chowdhury ${ }^{2 *}$, A. A. $\operatorname{Rana}^{1}$, S. M. Masum ${ }^{1}$, T. Ferdous ${ }^{1}$, M. A. Rashid ${ }^{2}$, \\ M. Sarker ${ }^{1}$ and M. M. Karim ${ }^{1 *}$ \\ ${ }^{1}$ Department of Applied Chemistry and Chemical Engineering, University of Dhaka, Dhaka-1000, Bangladesh. \\ ${ }^{2}$ Department of Pharmaceutical Chemistry, University of Dhaka, Dhaka-1000, Bangladesh.
}

\begin{abstract}
Microcrystalline cellulose (MCC) is an important ingredient in pharmaceutical, food, cosmetic and other industries. Microcrystalline cellulose was synthesized from the alpha cellulose content of pretreated cotton, Bombax ceiba L. by hydrochloric acid hydrolysis. The prepared microcrystalline cellulose was characterized by determining some physicochemical properties such as $\mathrm{pH}$, angle of response, Carr's index, Hausner ratio, moisture content etc and compared with commercial-grade microcrystalline cellulose that is used in pharmaceutical industry as excipient. Scanning electron microscope (SEM) and FTIR data represented the structure and particle characterization of sample. Thermal gravimetric analysis (TGA) showed the thermal stability of the sample. The results showed that the yield of microcrystalline cellulose was about $85 \%$ and compared favorably with the commercial grade microcrystalline cellulose as well as conformed official specifications for microcrystalline cellulose in British Pharmacopeia. It was also found that the duration of acid hydrolysis affected the polymeric form of the processed alpha cellulose.
\end{abstract}

Keywords: Bombax ceiba L.; Microcrystalline cellulose; Thermal gravimetric analysis; Scanning electron microscope

\section{Introduction}

The preparation of tablets using direct compression method has steadily increased due to the numerous advantages of direct compression technology in tableting. These include economy, elimination of granulation process, and uniformity of particle size and greater stability of tablets on aging (Shangraw, 1984). Currently, microcrystalline cellulose (MCC) is the most commonly used direct compression excipient (Setu et al., 2014). MCC is produced by reacting alpha cellulose with an aqueous solution of a strong mineral acid at boiling temperature for a period until the level-off degree of polymerization (level-off DP) of cellulose is obtained. MCC is not only highly compressible but also it increases compressibility of other excipients when added in small quantities. It is an effective dry binder in low concentration. It has sufficient fluidity to be directly compressed and to exhibit disintegrating properties (Bolhuis et al., 1996). Commercially available MCC is derived from both gymnosperms (generally conifers) and other softwoods, and from hardwood dicotyledons and cotton. These differ considerably in chemical composition (proportions of cellulose, hemicelluloses, and lignin) and structural organization which affect the composition of the $\alpha$-cellulose extracted and the composition and crystallinity of MCC finally produced (Landin et al., 1993). Besides the wood pulp as a source of cellulose and its derivatives, the purified cotton linters obtained from Bombax ceiba L. are also a common source (Evans, 1989). In Bangladesh, Bombax ceiba L. locally known as shimul-tula is found everywhere in the country especially northern area of Bangladesh where its availability is the highest. The use of alternative non-wood sources of fiber in preparation of pulp for industrial applications has received substantial attention. There are two important reasons for the continuous increase in this area. Firstly, there is a decrease in wood availability with the increasing demand for market pulp in some rapidly developing countries in Asia, Africa, and Latin America. Secondly, silk cotton are excellent alternative materials to substitute wood because they are plentiful, widespread, and easily accessible. Aside from their abundance and renewability, utilization of silk cotton has advantages for economy, environment, and technology. The microcrystalline cellulose (MCC) is a natural cellulosic polymer composed of D-glucose monomer units bonded with 1, 4- linkage. The definition microcrystalline is related to the order of the cellulose chains in the natural fiber. Regions with

\footnotetext{
*Corresponding author: E-mail: moinulcd@yahoo.com, asaddg27@du.ac.bd
} 
higher degree of order are called microcrystalline; the other regions with low degree of order are called amorphous. The natural cellulose contains about $40-45 \%$ microcrystalline and $55-60 \%$ amorphous part. The isolation of the MCC is made by an acidic hydrolysis with $\mathrm{HCl} / \mathrm{H}_{2} \mathrm{O}$ at high temperatures (Dong et al., 1998). Acid hydrolysis is effective in dissolving the amorphous cellulose, leaving behind the micro-crystalline cellulose. The yields of micro-crystalline cellulose from these raw materials are high. The acid hydrolysis of cellulose produces mainly glucose from the amorphous part (Jahan et. al., 2011). It is well accepted that powder properties of the micro-crystalline cellulose are vital in tablet formulation (Sakurada et. al., 1962) and compressibility and binding properties are increased by using this method.

Traditionally, MCC has been prepared from bamboo (Sun et. al., 2008), wood pulp (Durand et al., 1970) and viscose rayon (Battista et. al., 1962). Attempts have also been made to produce MCC from other sources such as newsprint waste (Bhimte et al., 2007), and corncobs (Nagavi et. al., 1989), as well as from fast-growing plants including sesbania sesban, sroxburghii, crotalaria juncea (Jain et. al., 1983), bagasse, rice straw (El-Sakhaw et. al., 2007). In recent years, there has been enormous interest in producing MCC for pharmaceutical use because of its high compactness under minimum compression pressures, high binding capability, and ability to prepare tablets that are extremely hard, stable, yet disintegrate rapidly (Dittgen et. al., 1993; Doelker et. al., 1987). Report on preparation of microcrystalline cellulose from cotton rags has been published earlier (Yuvraj et. al., 2009). No data has been published in the preparation of MCC from cotton of Bombax ceiba L. available in Bangladesh. The purpose of the present study is to prepare MCC from Bombax ceiba L. and its physicochemical evaluation of different parameters to check the possibility of prepared MCC to use as pharmaceutical excipient.

\section{Materials and methods}

\section{Materials and instruments}

Raw material from Bombax ceiba was collected from Mymensingh in Bangladesh. It was further chemically treated and prepared for alpha cellulose. Analytical grade chemicals used in this experiment include hydrochloric acid $(\mathrm{HCl}$, Merck, Germany), sodium carbonate $\left(\mathrm{Na}_{2} \mathrm{CO}_{3}\right.$, Merck, Germany), buffer solution $\left(\mathrm{CH}_{3} \mathrm{COOH}+\mathrm{CH}_{3} \mathrm{COONa}\right.$ Solution, Merck, Germany), distilled water, $\mathrm{H}_{2} \mathrm{O}$, sodium hydroxide ( $\mathrm{NaOH}$, Merck, Germany), methyl orange $\mathrm{BDH}$, UK and instruments used to characterize the microcrystalline cellulose include electrical balance (SPB 31, Kaifeng Group Co.Ltd.), ostwald's viscometer (Ernst Haage), hot plate stirrer (Thermolyne Mirak), electric wall oven (temperature range up to $450^{\circ} \mathrm{C}$, EOEM61AS, Electrolux), FTIR spectrophotometer, (measuring range: $400-4000 \mathrm{~cm}^{-1}$, Shimdazu), thermo gravimetric analyzer (measuring temperature range: ambient to $1500^{\circ}$, Rigaku).

\section{Procedure}

Microcrystalline cellulose was prepared from alpha cellulose of pre-treated cotton by acid hydrolysis (Dong et al., 1998). Commercial grade standard microcrystalline cellulose was collected from market and named as sample MCC-1 (Standard). $10 \mathrm{~g}$ of the alpha cellulose obtained from pretreated cotton was placed in pyrex glass beaker and hydrolyzed with $5 \mathrm{~N}, 4 \mathrm{~N}, 3 \mathrm{~N}, 2 \mathrm{~N}, 1 \mathrm{~N}$ of $200 \mathrm{ml}$ hydrochloric acid. The alpha cellulose was soaked with a spatula at room temperature for $30 \mathrm{~min}$. Then acid soaked alpha cellulose mixture was heated at $100^{\circ} \mathrm{C}$ for one hour. Vigorous stirring with a spatula was done at the time of heating. After one hour heating and stirring the sample was cooled at room temperature. The microcrystalline cellulose obtained by this process was washed with water until total hydrochloric acid was removed and then pressed. The sample was dried in a fluidized bed dryer at an inlet air temperature of $60-65^{\circ} \mathrm{C}$ for overnight. Following further milling and sieving, the fraction passing through $0.710 \mathrm{~mm}$ sieve was obtained and stored at room temperature in desiccators and yields of MCC were $81.8 \%, 83.9 \%, 85.5 \%, 86.4 \%$ and $88.61 \%$ respectively. Different properties such as intrinsic viscosity, moisture content, $\mathrm{pH}$, angle of repose, bulk and tapping densities, Carr's index and Hausner ratio, three-dimensional appearance by SEM, absorption band spectra by FTIR and TGA of various samples of MCC were investigated and compared with MCC-1 (standard).

\section{Results and discussion}

The yield of the prepared microcrystalline cellulose samples obtained from chemically modified cotton was approximately $85 \% \mathrm{w} / \mathrm{w}$. The results of the physicochemical analyses indicate a high level of purity of the cellulose material. The organoleptic properties of the microcrystalline cellulose samples produced were good as the material was odourless, tasteless, white and granular in texture. The characteristics of microcrystalline cellulose samples were discussed below.

\section{Moisture content}

The moisture content of prepared microcrystalline cellulose samples as shown in the Figure 1 were about 5 to $6 \%$ which is well below the official limit of $8 \%$ stated in British Pharmacopoeia, 1993. This low value is indicative of the suitability of microcrystalline cellulose from cotton as diluents in the formulation of hydrolysable drugs such as aspirin. 


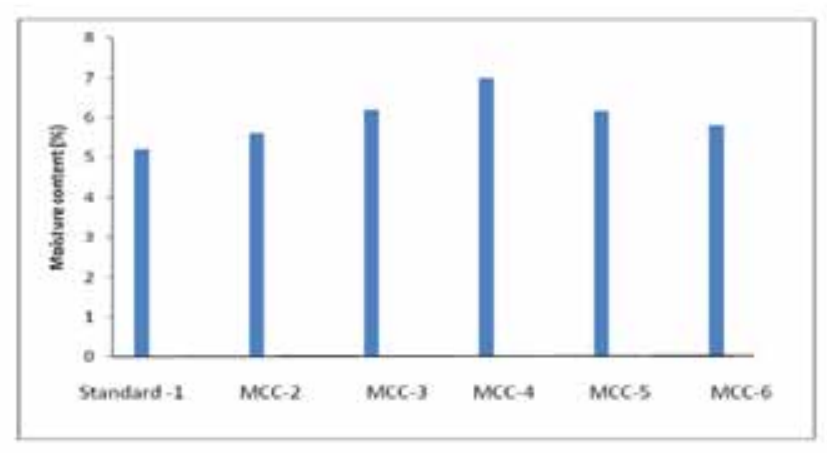

Fig. 1. Moisture content of the MCCs

\section{pH of cellulose solution}

According to British Pharmacopeia $\mathrm{pH}$ of microcrystalline cellulose solution should be 5-7. The $\mathrm{pH}$ of the prepared microcrystalline cellulose samples were about 6 to 7 as shown in the Figure 2 which is within the acceptable range of standard microcrystalline cellulose. From the Figure it is observed that the standard commercial grade microcrystalline cellulose sample that is used in pharmaceutical industry in Bangladesh as excipient, shows similar $\mathrm{pH}$ to the prepared microcrystalline cellulose samples.

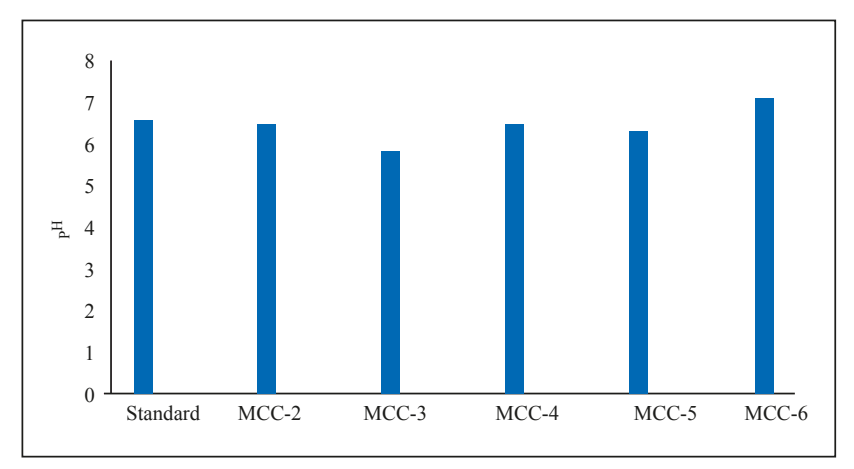

Fig. 2. pH of the samples solution

\section{Carr's Index and Hausner Ratio}

Carr's compressibility indexes of the samples are shown in the Figure 3. From the Figure it can be seen that only the sample MCC-2 has almost similar Carr's compressibility index to standard microcrystalline cellulose sample but the rest of the samples have very high indices. The flow properties of powder are essential in determining the suitability of a material as a direct compression excipient. Hausner ratio and Carr's compressibility index are considered as indirect measurements of powder flowability (Staniforth, 1996). Again the Hausner ratio is indicative of interparticle friction, and the Carr's index shows the aptitude of a material to diminish in volume. As the values of these indices from samples MCC-3 to MCC-6 increase, their flowability decreases. In general however, Hausner ratio greater than 1.25 indicates poor flow and Carr's compressibility index below $16 \%$ indicates good flowability while values above $35 \%$ indicate cohesiveness.

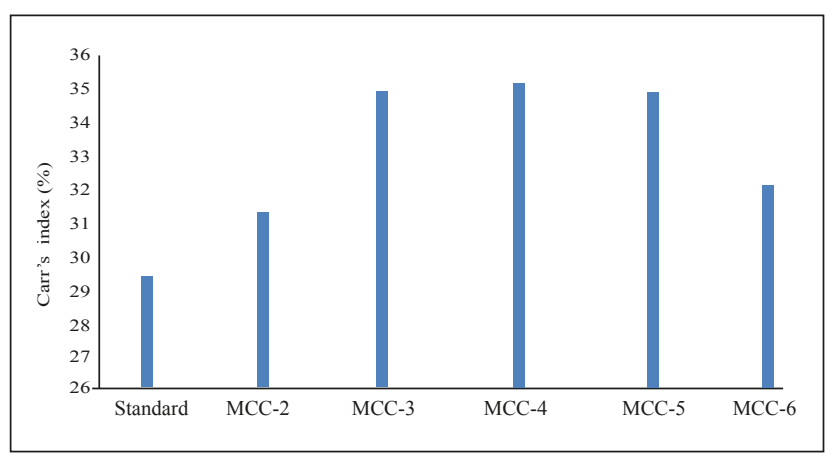

Fig. 3. Carr's compressibility index of the samples

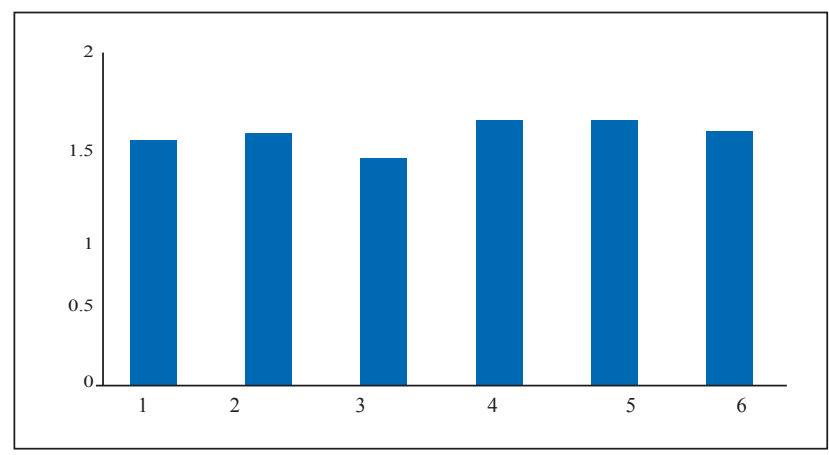

Fig. 4. Hausner ration of the MCC sample. 1, 2, 3, 4, 5 and 6 indicates standard, MCC-2, MCC-3, MCC-4, MCC-5 and MCC-6 respectively. $\mathrm{Y}$-axis indicates the Hausner ratio value

Angle of Repose

The angle of repose gives a qualitative assessment of internal and cohesive frictional forces. An angle $<30^{\circ}$ indicates good flow potential, while an angle of $>40^{\circ}$ exhibits poor or absence of flow (Banker et al., 1987). All prepared microcrystalline cellulose samples, MCC-2, MCC-3, MCC-4, MCC-5 and MCC-6 showed an angle of repose < $40^{\circ}$ as shown in the Figure 5 and were therefore classified as materials with reasonable flow potential. MCC-2, MCC-3 
and MCC-4 showed good flow property compared to the standard making the prepared MCC a promising candidate for use as tablet excipient.

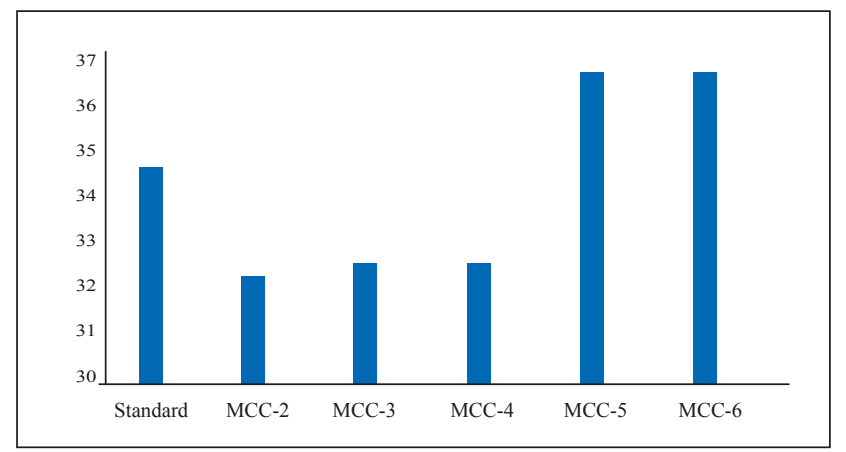

Fig. 5. Angle of repose of the samples

\section{Scanning electron microscopy}

Figure 6 compares the SEM photographs of commercial grade standard micro crystalline cellulose and prepared MCCs (MCC-2 to MCC-6). All MCCs consist of non-aggregated fibers. There is no difference between them except for short fibers seen in MCC-3 and MCC-4. The variation of particle shape might be due to variation of preparation method of the samples.

\section{FTIR analysis}

The FTIR spectra of commercial grade standard microcrystalline cellulose and experimental samples are compared. These spectra are similar. The Figure 7 shows spectra of standard MCC and a typical MCC-3. The analysis of the spectra with reference to published data show several typical features of the cellulose which include: (i) the characteristic intermolecular and intramolecular $\mathrm{OH}$ stretching vibration band in the spectra which occur at 3345 , $3342.64,3342.64,3342.64,3350$ and $3344.57 \mathrm{~cm}^{-1}$, respectively, for standard grade microcrystalline cellulose and MCC-2, MCC-3, MCC-4, MCC-5 and MCC-6; (ii) the peak at 1433,1367 , and $1325 \mathrm{~cm}^{-1}$ are associated with intermolecular hydrogen bonds at the $\mathrm{C}$ group and the $\mathrm{OH}$ in plane bending vibration, respectively. (iii) As like as in the spectrum of the standard, the absence of prominent peak at $1737 \mathrm{~cm}^{-1}$ in the extracted cellulose indicated that chemical treatment effectively removed lignin and hemicelluloses from the raw materials. A peak at this position indicates

either the acetyl and uronic ester groups of the hemicelluloses or the ester linkage of carboxylic group of the ferulic and $p$-coumeric acids of lignin and/or hemicelluloses (Sonaglio et. al., 1995; Chatchawalsaisin et. al., 2005).

The lack of peak at $1513 \mathrm{~cm}^{-1}$, indicative of the aromatic ring lignin is attributed to the effective removal of lignin during

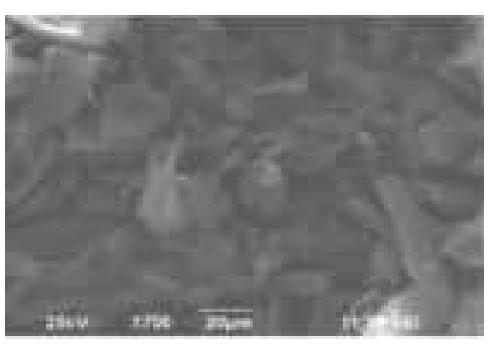

STANDARD (Standard)

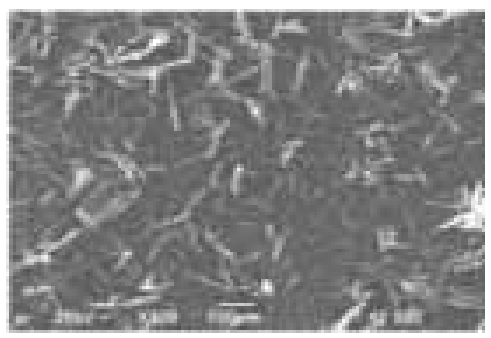

MCC-4

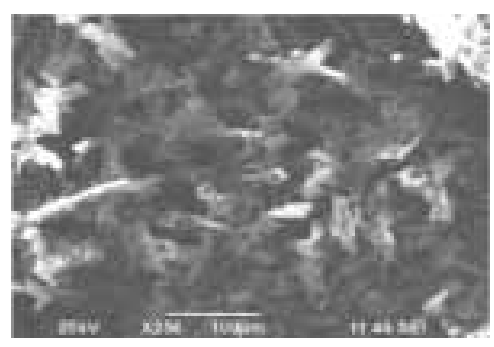

MCC-2

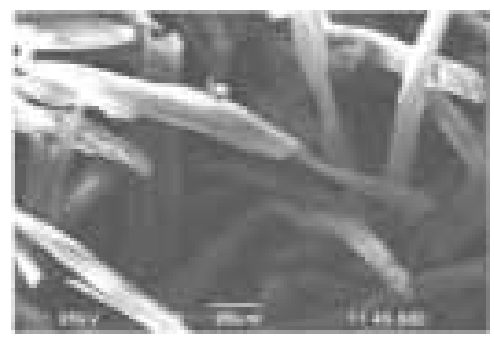

MCC-5

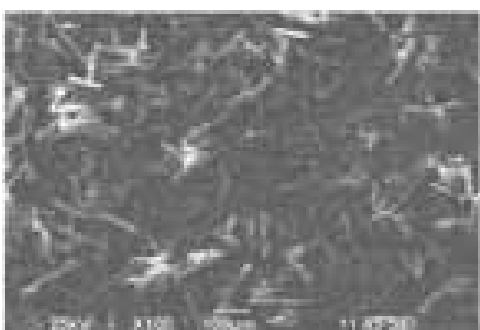

MCC-3

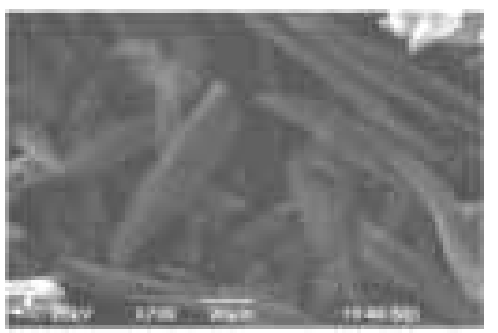

MCC-6

Fig. 6. SEM images of the standard and sample 
treatment. The acid hydrolysis removed the amorphous cellulose on the surface, therefore, more $\mathrm{C}-\mathrm{OH}, \mathrm{C}-\mathrm{O}-\mathrm{C}$ and $\mathrm{C}-\mathrm{C}$ bonds were exposed, resulting in the increases in the stretching absorbency (Sun et. al., 2008, Sun et. al., 2007).

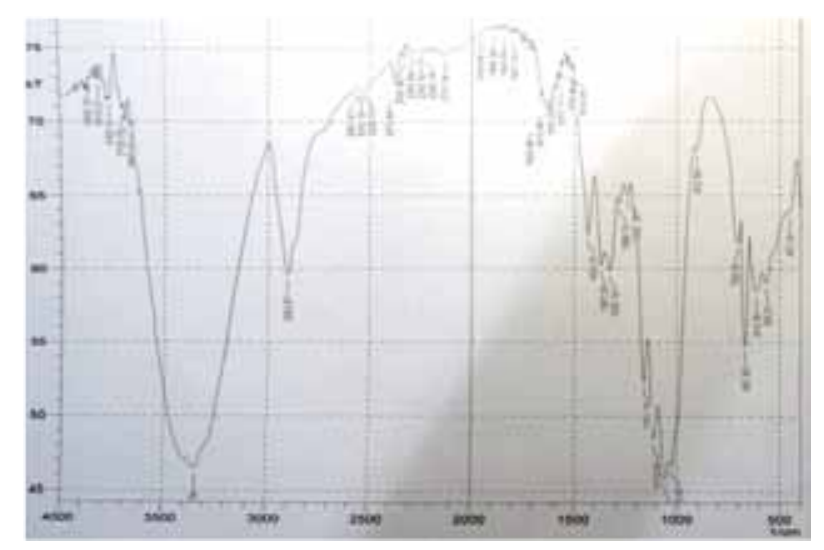

(1)

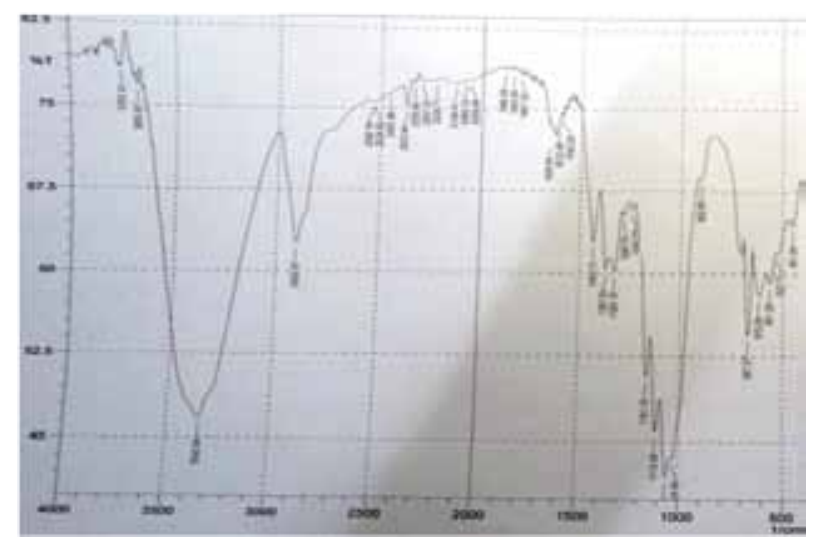

(2)

\section{Thermogravimetric analysis}

The thermal properties of MCC are important for production of tablet. TG curves of MCCs obtained from cotton are shown in the Figure 8. Initial weight loss was observed from 50 to $150^{\circ} \mathrm{C}$ for all samples due to evaporation of moisture. The degradation behaviors of all micro crystalline cellulose samples were almost similar, started above $300^{\circ} \mathrm{C}$. The high residue of MCC-2 was due to high silica content.

The sample MCC-6 showed almost similar degradation temperature, but lower residue. The degradation peaks and residues for MCC-3 and MCC- 5 are 368 and $353^{\circ} \mathrm{C}$ respectively. It can is seen that the cellulose decomposition started at $315^{\circ} \mathrm{C}$ and continued until $400^{\circ} \mathrm{C}$. The maximum



Fig. 8. Thermal degradation behaviors of MCCs and standard

weight loss rate was reached at $355^{\circ} \mathrm{C}$. Based on above results, it can be concluded that the microfibril samples from cotton have good thermal stability at $300^{\circ} \mathrm{C}$ and can be suitable in the production of tablet.

\section{Conclusion}

MCCs prepared in this work from cotton collected from Bombax ceiba, have properties similar to that of a standard grade commercial MCC. The FTIR is same for prepared and commercial MCC. The TGA curve of the prepared and commercial $\mathrm{MCC}$ are very similar. Initial degradation temperature of the prepared MCCs shows that they have good thermal stability. The total weight loss found at $600^{\circ} \mathrm{C}$ is found $99.41 \%$ : which shows that only $0.59 \%$ is the residue present in the prepared MCC. These results indicate similar crystallinity for both MCC samples i.e. for commercial and prepared from cotton. So cotton collected from Bombax ceiba $L$. may be an interesting alternative as cellulose source for several applications. It can reduce the rate of import of microcrystalline cellulose used for several applications which can effectively save the foreign currency. But before using these microcrystalline cellulose as an excipients in tablet formulation, further investigations such as disintegration test, friability test, side effect when oral consumption, must be studied.

\section{References}

Banker GS and Anderson NR (1987), In: The Theory and Practice of Industrial Pharmacy, third ed. Lachman L, Lieberman HA, Kanig JL, editors. Varghese Publishing House; Mumbai: 293-299. 
Battista OA and Smith PA (1962), Microcrystalline cellulose. Indust. and Eng. Chem., 54: 20-29.

Bhimte NA and Tayade PT (2007), Evaluation of microcrystalline cellulose prepared from sisal fibers as a tablet excipient: A technical note. AAPS Pharm. Sci. \& Technol., 8: 1-6.

Bolhuis GK and Chowhan ZT (1996), Materials for direct compaction. In: Alderborn G, Nystrom C, editors. Pharmaceutical Powder Compaction Technology. NewYork: Merkcel Dekker Inc : 419-500.

Chatchawalsaisin J, Podczeck F and Newton JM (2005), The preparation by extrusion/spheronization and the properties of pellets containing drugs, microcrystalline cellulose and glyceryl monostearate, Eur J Pharm Sci, 24: $35-48$.

Dittgen M and Fricke S (1993), Microcrystalline cellulose in direct tableting. Mfg Chem. 64(7) 17-21.

Doelker E, Mordier D, Iten H and Humbert-Droz P (1987), Comparative tableting properties of sixteen microcrystalline cellulose. Drug dev Ind Pharm. 13: 1847-1875.

Dong XM, Revol JF and Gray DG (1998), Effect of microcrystalline preparation conditions on the formation of colloid crystals of cellulose, Cellulose, 5 (1): 19-32.

Durand HW, Fleck EJ and Raynor GE (1970), Microcrystalline cellulose compositions Co-dried with hydrocelluloses. US patent. 3: 537-058, USA.

El-Sakhawy M and Hassan ML (2007), Physical and mechanical properties of microcrystalline cellulose prepared from agricultural residues. Carbohyd. Poly., 67: $1-10$.

Evans WC (1989), Trease and Evans' Pharmacognosy. 13th ed. Bailliere Tindall: 339-77.

Jahan M, Saeed A, He Z and Ni Y (2011), Jute as raw material for preparation of microcrystalline cellulose, Cellulose, 18(2): 451-459.

Jain AK, Dixit VK and Varma KC (1983), Preparation of microcrystalline cellulose from cereal straw and its evaluation as a tablet excipient. Indust. J. Pharm. Sci., 3: $83-85$.

Landin M, Martinez-Pacheco R, Gomez-Amoza JL, Souto C, Concheiro A and Rowe RC (1993), Effect of batch variation and source of pulp on the properties of microcrystalline cellulose, Int J Pharm, 91: 133-41.

Nagavi BG, Mithal BM and Chawla JS (1989), Microcrystalline cellulose from corncobs. Research Industry, 28: 277-280.

Sakurada I, Nukushina Y and Ito T (1962), Experimental Determination of Elastic Modulus of Crystalline Regions in Oriented Polymers, Journal of Polymer Science, 57(165): 651-660.

Setu MNI, Mia MY, Lubna NJ and Chowdhury AA (2014), Preparation of Microcrystalline Cellulose from Cotton and its Evaluation as Direct Compressible Excipient in the Formulation of Naproxen Tablets, Dhaka University Journal of Pharmaceutical Sciences, 13(2): 187-192.

Shangraw RF (1984), Advantages and disadvantages of wet granulation and direct compression processes for making tablets. Modern Granulation, Tableting and Capsule Technology, Centre for Professional Advancement. Amsterdam, Netherlands.

Sonaglio D, Battaille B, Ortigosa C and Jacob M (1995), Factorial design in the feasibility of producing Microcel MC 101 pellets by extrusion/spheronization, Int J Pharm, 115: 53-60.

Staniforth JN (1996), Powder flow. In: Aulton ME, editor. Pharmaceutics - The Science of Dosage form Design. London, Churchill Livingston: 600-15.

Sun Y, Lin L, Deng H, LJ, HeB, Sun R and Ouyang P (2008), Structural changes of bamboo cellulose in formic acid, BioResources, 3(2): 297-315.

Sun Y, Lin L, Pang C, Deng H, Peng H and Li J, HeB and Lius $S$ (2007), Hydrolysis of Cotton fiber cellulose in formic acid, Energy and Fuels, 21: 2386-2389.

Yuvraj C, Sapkal RS, Sakpal VS and Zamre GS (2009), Microcrystalline cellulose from waste cotton rags (waste from garments and hosiery industries). Hydrogels for medical applications fabricated by oxidative-hydrolytic modification of cellulose. Int. J. Chem. Sci.: 7(2): 681-688.

Received: 11 August 2015; Revised: 15 October 2015; Accepted: 19 October 2015. 